\title{
Article
}

\section{Weaker form of totally continuous functions}

\author{
Md. Hanif Page ${ }^{1}$ and Lakshmi Narayan Mishra ${ }^{2, *}$ \\ 1 Department of Mathematics, KLE Technological University, Hubballi-580031, Karnataka, India; hanif01@yahoo.com \\ 2 Department of Mathematics, School of Advanced Sciences, Vellore Institute of Technology (VIT) University, Vellore \\ 632 014, Tamil Nadu, India) \\ * Correspondence: lakshminarayanmishra04@gmail.com
}

Received: 04 August 2018; Accepted: 10 December 2018; Published: 8 February 2019.

\begin{abstract}
The objective of this paper is to study new type of continuous functions called totally $\alpha$ gs-continuous functions using $\alpha$ gs-open sets. Furthermore we discuss covering properties and obtain their characterizations by including counter examples.
\end{abstract}

Keywords: Totally $\alpha$ gs-continuous, $\alpha$ gs-closed set, $\alpha$ gs-continuous, $\alpha$ gs-connected.

MSC: 54C08, 54C10.

\section{Introduction}

G eneral topology plays an important role in many fields of applied sciences as well as branches of mathematics. In general topology, generalized open sets plays an important role. Indeed a significant theme in general topology and real analysis concerns that variously modified forms of continuity, separation axioms etc. by utilizing generalized open sets.

Continuous functions stand among the most fundamental concepts in the whole mathematical science. Many different forms of stronger and weaker forms of such functions have been introduced and studied over the years in the field of general topology. The idea of totally continuous functions presented in 1980 by Jain [1]. In [2] totally semi continuous functions presented as a generalization of totally continuous functions and basic results were proved. Recently, Rajamni and Vishwanathan [3] introduced the notion of $\alpha$ gs-closed set using $\alpha$-closure operator. Using $\alpha$ gs-closed set, almost contra $\alpha$ gs-continuous as well as contra ags-continuous functions were introduced in [4].

In this paper the new generalization of total continuity named as totally $\alpha$ gs-continuous is presented. The notion of totally $\alpha$ gs-continuous is a weaker form of total continuity. Also it's fundamental properties are investigated.

\section{Preliminaries}

In the entire paper $(X, \tau),(Y, \sigma)$ (or simply $X, Y)$ represents topological spaces on which no separation axioms are assumed unless explicitly stated. $\operatorname{Cl}(A)$ and $\operatorname{Int}(A)$ represents closure and interior of $A$ with respect to $\tau$ in the sequel. We recall some definitions which are necessary.

Definition 1. A subset $A$ of a topological space $X$ is known as:

1. semi open set [5] if $A \subset C l(\operatorname{Int}(A))$.

2. semi closed set [6] if $\operatorname{Int}(\operatorname{Cl}(\operatorname{Int}(A))) \subset A$.

3. $\alpha$-open [7] if $A \subset \operatorname{Int}(\operatorname{Cl}(\operatorname{Int}(A)))$.

Definition 2. [3] A subset $A$ of $X$ is $\alpha$ generalized semi-closed (shortly, $\alpha$ gs-closed) set if $\alpha \operatorname{Cl}(A) \subset U$ whenever $A \subset U$ and $U$ is semi open in $X$.

The complement of $\alpha$ gs-closed set is $\alpha$ generalized-semi open (shortly, $\alpha$ gs-open). The family of all $\alpha$ gs-closed sets of $X$ is denoted as $\alpha \mathrm{GSC}(X, \tau)$ besides $\alpha$ gs-open sets by $\alpha \mathrm{GSO}(\mathrm{X}, \tau)$.

Definition 3. [8] The intersection of all $\alpha$ gs-closed sets containing a set $A$ is said to be $\alpha$ gs-closure of $A$ and is denoted by $\alpha \operatorname{gsCl}(A)$. A set $A$ is $\alpha$ gs-closed if and only if $\alpha \operatorname{gsCl}(A)=A$. 
Definition 4. [8] The union of all $\alpha$ gs-open sets contained in $A$ is said to be $\alpha$ gs-interior of $A$ and is denoted by $\alpha \operatorname{gs} \operatorname{Int}(A)$. A set $A$ is $\alpha \mathrm{gs}$-open if and only if $\alpha \operatorname{gs} \operatorname{Int}(A)=A$.

Definition 5. [9] A topological space $X$ is said to be $\alpha g s-T_{2}$ if for each pair of distinct points $x$ and $y$ of $X$, there exists disjoint $\alpha$ gs-open sets, one containing $x$ and the other containing $y$.

Definition 6. [1] A function $h: X \rightarrow Y$ is said to be totally continuous if the inverse image of each open subset of $Y$ is a clopen subset of $X$.

Definition 7. [4] A topological space $X$ is said to be $\alpha$ gs-connected if it cannot be written as the union of two non-empty disjoint $\alpha$ gs-open sets.

\section{Totally $\alpha$ gs-Continuous Functions}

In this section, the idea of a new class of functions named totally $\alpha$ gs-continuous function is presented. In addition, the relationships with existing functions are discussed.

Definition 8. A function $\mu: X \rightarrow Y$ is said to be totally $\alpha$ gs-continuous if $\mu^{-1}(K)$ is $\alpha$ gs-clopen in $X$ for every open set $K$ of $Y$.

Remark 1. Every totally continuous function is totally $\alpha$ gs-continuous. The converse may not be true.

Example 1. Consider topologies $\tau=\{X, \phi,\{p\},\{q, r\}\}, \sigma=\{Y, \phi,\{p\},\{p, q\}\}$ on $X=Y=\{p, q, r\}$, We have $\alpha \operatorname{GSC}(X)=\{\{q\},\{r\},\{p, q\},\{p, r\}\}$. Define a function $h: X \rightarrow Y$ by $h(p)=q, h(q)=p, h(r)=r$. Then $h$ is totally $\alpha$ gs-continuous but it is not totally continuous as $h^{-1}(\{p\})=\{q\}$ is not clopen in $X$.

Remark 2. Every totally $\alpha$ gs-continuous is $\alpha$ gs-continuous function. But the converse is not true as illustrated by the following example.

Example 2. Let $X=\{a, b, c\}, \tau=\{X, \phi,\{a\},\{a, b\}\}$. We have $\alpha \operatorname{GSC}(X)$ are $\{\{c\},\{b, c\}\}$. Let $Y=\{1,2,3\}$, $\sigma=\{Y, \phi,\{1,2\}\}$. Define a function $h: X \rightarrow Y$ by $\mathrm{h}(\mathrm{a})=2, \mathrm{f}(\mathrm{b})=1, \mathrm{f}(\mathrm{c})=3$. Then $\mathrm{h}$ is totally $\alpha$ gs-continuous but it is not $\alpha$ gs-continuous as $h^{-1}(\{1,2\})=\{a, b\}$ is $\alpha$ gs-open set but not $\alpha$ gs-closed set in $X$.

Theorem 9. If $f$ is totally $\alpha g s$-continuous function from $\alpha g$ s-connected space $X$ onto any space $Y$, then $Y$ is an indiscrete space.

Proof. Assume that $Y$ isn't indiscrete. Let $K$ be a proper non-empty subset of $Y$. Then $f^{-1}(K)$ is non-empty $\alpha$ gs-clopen subset of $X$, which is contradiction to the fact that $X$ is $\alpha$ gs-connected space. Then $Y$ is indiscrete space.

Theorem 10. Let $X$ be $\alpha g s$-connected with $Y$ be $T_{1}$. If $h: X \rightarrow Y$ is totally ags-continuous, then $f$ is constant.

Proof. Let $X$ be a $\alpha$ gs-connected and $h$ as totally $\alpha$ gs-continuous. Since $Y$ is $T_{1}$-space, $\Lambda=\left\{h^{-1}(y): y \in Y\right\}$ is a disjoint $\alpha$ gs-clopen partition of $X$. If $|\Lambda| \geq 2$, then there exists a proper $\alpha$ gs-clopen set $M$ for some $U \in \Lambda$ in $\alpha$ gs-connected space $X$. This is contradiction to the fact that $X$ is $\alpha$ gs-connected. So $|\Lambda|=1$. Therefore $f$ is constant.

Definition 11. Consider a topological space $X$. We define an equivalence relation on $X$ by taking $p \approx q$ if there is a $\alpha$ gs-connected subset of $X$ containing $p$ as well as $q$. The equivalence families are said to be $\alpha$ gs-separation of $X$ or $\alpha$ gs-component of $X$.

Theorem 12. Let $h: X \rightarrow Y$ be a totally $\alpha$ gs-continuous function from a topological space $X$ into $T_{1}$ space $Y$. Then $h$ is constant on each $\alpha$ gs-component of $X$.

Proof. This result follows immediately from above theorem. 
Definition 13. For a subset $A$ of $X, \alpha \operatorname{gsCl}(A)-\theta \operatorname{gs} \operatorname{Int}(A)$ is said to be $\alpha$ gs-frontier of $A$ and is denoted as $\alpha \operatorname{gs}-\operatorname{Fr}(A)$.

Theorem 14. The set of all points $p$ of $X$ at which $h: X \rightarrow Y$ is not totally ags-continuous is identical with the union of ags-frontier if the inverse images of closed sets of $Y$ containing $h(x)$.

Proof. Assume that $h$ is not totally $\alpha$ gs-continuous at $p \in X$, then there exists an open set $V$ of $Y$ containing $h(x)$ such that $h(U)$ is not contained in $V$ for each $\alpha$ gs-clopen set $U$ containing $p$. This indicates $U \cap\left(X-h^{-1}(V)\right) \neq \phi$ for each $U \in \alpha G S O(X, p)$. Therefore, $p \in \alpha g s C l\left(X-h^{-1}(V)\right)$. But, as $p \in h^{-1}(V) \subset$ $\alpha \operatorname{gsCl}\left(h^{-1}(V)\right), p \in \alpha \operatorname{gsCl}\left(h^{-1}(V)\right) \cap \alpha \operatorname{gsCl}\left(X-h^{-1}(V)\right)$. This shows that, $p \in \alpha g s-F r\left(h^{-1}(V)\right)$.

Conversely, assume $p \in \alpha g s-F r\left(h^{-1}(V)\right)$ for some open set $V$ of $Y$ containing $h(x)$ with $h$ is totally $\alpha$ gs-continuous at $p \in X$. Then there exists $U \in \alpha G S O(X, p)$ such that $h(U) \subset V$. This indicates $x \in U \subset h^{-1}(V)$. Therefore $p \in \alpha g s \operatorname{Int}\left(h^{-1}(V)\right) \subset X-\alpha g s-F r\left(h^{-1}(F)\right)$. This is contradiction to the fact that, $x \in \alpha g s-F r\left(h^{-1}(F)\right)$. Therefore $h$ is not totally $\alpha$ gs-continuous.

Definition 15. A filter base $\Omega$ in a topological space $X$ is said to be $\alpha$ gs-co-convergent to a point $q$ in $X$ if for any $U \in \alpha G S O(X)$ containing $q$, there exists $K \in \Omega$ such that $K \subset U$.

Theorem 16. If a function $h: X \rightarrow Y$ is totally $\alpha g s$-continuous, then for each point $q \in X$ and every filter base $\Omega$ in $X$ ags-co-converging to $q$, therefore filter base $h(\Omega)$ is convergent to $h(q)$.

Proof. Let $q \in X$ as well as $\Omega$ be any filter base in $\alpha$ gs-co-converging to $q$. Since $h$ is totally $\alpha$ gs-continuous, then for any open set $V$ of $Y$ containing $h(q)$, there exists $U \in \alpha G S O(X)$ containing $q$ such that $h(U) \subset V$. As $\Omega$ is $\alpha$ gs-co-converging to $q$, there exists $K \in \Omega$ such that $K \subset U$. This means that $h(K) \subset V$ and therefore the filter base $h(\Omega)$ is convergent to $h(q)$.

\section{Covering Properties}

Definition 17. A topological space $X$ is said to be:

1. $\alpha$ gs-co-compact if every $\alpha$ gs-clopen cover of $X$ has finite subcover.

2. $\alpha$ gs-co-compact relative to $X$ if every cover of $A$ by $\alpha$ gs-clpoen sets of $X$ has finite subcover.

3. $\alpha$ gs-compact [4] if every $\alpha$ gs-open cover of $X$ has finite subcover.

4. $\alpha$ gs-compact [4] if $A$ is a $\alpha$ gs-compact as a subspace of $X$.

Theorem 18. If a function $\mu: X \rightarrow Y$ is totally $\alpha g s-c o n t i n u o u s$ as well as $Q$ is $\alpha g s$-co-compact relative to $X$, then $\mu(Q)$ is compact in $Y$.

Proof. Let $\left\{H_{i}: i \in I\right\}$ be any cover of $\mu(Q)$ by open sets of $Y$. For each $x \in Q$, there exists $i_{x} \in I$ such that $\mu(x) \in\left(H_{\alpha}\right)_{x}$ and there exists $U_{x} \in \alpha G S O(X)$ containing $x$ such that $\mu\left(U_{x}\right) \subset\left(H_{\alpha}\right)_{x}$. Since the collection $\left\{U_{x}: x \in Q\right\}$ is a cover of $Q$ by ags-clopen sets of $X$, there exists a finite subset $Q_{0}$ of $Q$ such that $Q \subset \cup\left\{U_{x}: x \in Q_{0}\right\}$. So, we get $\mu(Q) \subset \cup\left\{\mu\left(U_{x}\right): x \in Q_{0}\right\} \subset \cap\left\{\left(H_{\alpha}\right)_{x}: x \in Q_{0}\right\}$ and hence $\mu(Q)$ is compact.

Theorem 19. Following results are equivalent for a function $h: X \rightarrow Y$

1. $h$ is totally ags-continuous,

2. for every open set $V$ of $Y, h^{-1}(V)$ is ags-clopen in $X$,

3. for every closed set $F$ of $Y, h^{-1}(F)$ is ags-clopen in $X$.

Proof. $\mathbf{1} \Rightarrow \mathbf{2}$ Consider an open set $V$ in $Y$ as well as $x \in h^{-1}(V)$. Then $h(x) \in V$. By (1), there exists a $\alpha$ gs-clopen set $U_{x}$ in $X$ such that $x \in U_{x} \subset h^{-1}(V)$. This implies $h^{-1}(V)$ is $\alpha$ gs-clopen nhd of $x$. As $x$ is arbitrary, $h^{-1}(V)$ is $\alpha$ gs-clopen nhd of each of its points. This indicates $h^{-1}(V)$ is $\alpha$ gs-clopen in $X$.

$\mathbf{2} \Rightarrow \mathbf{1}$ Let $H$ be any open set in $Y$ containing $h(x)$, such that $x \in h^{-1}(H)$. Again by (2), $h^{-1}(H)$ is $\alpha$ gs-clopen in $X$. Set $G=f^{-1}(H)$, then $G$ is $\alpha$ gs-clopen set in $X$ containing $x$ with $h(G)=h\left(h^{-1}(H)\right) \subset H$. This implies $h$ is totally $\alpha$ gs-continuous at $x \in X$. As $x$ is arbitrary, it follows that $h$ is totally $\alpha$ gs-continuous at each point $x$ of $X$. Then, $h$ is totally $\alpha$ gs-continuous.

$\mathbf{2} \Rightarrow \mathbf{3}$ Let $F$ be a closed set in $Y$. Then $Y-F$ is an open set in $Y$. By (2), $h^{-1}(Y-F)=X-h^{-1}(F)$ is $\alpha$ gs-clopen 
in $X$. This means $h^{-1}(F)$ is $\alpha$ gs-clopen in $X$.

$\mathbf{3} \Rightarrow \mathbf{2}$ Consider $O$ be an open set in $Y$, then $Y-O$ is a closed set in $Y$. By (iii), $h^{-1}(Y-O)=X-h^{-1}(O)$ is $\alpha$ gs-clopen in $X$. This implies $h^{-1}(O)$ is $\alpha$ gs-clopen in $X$.

Theorem 20. If $f: X \rightarrow Y$ is totally $\alpha g s-c o n t i n u o u s$ injection and $Y$ is $T_{0}$ then $X$ is $\alpha g s-T_{2}$.

Proof. Let $p$ and $q$ be any two distinct points in $X$. As $f$ is injective, $f(p)$ as well as $f(q)$ are distinct points in $Y$. As $Y$ is $T_{0}$, there exist an open set $U$ containing say $f(p)$ but not $f(q)$, which implies, $p \in f^{-1}(U)$ and $q \notin f^{-1}(U)$. As $f$ is totally $\alpha$ gs-continuous, $f^{-1}(U)$ is a $\alpha$ gs-clopen subset of $X$. Thus for two distinct points $p$ and $q$ of $X$, there exist two disjoint $\alpha$ gs-clopen subsets of $X$ such that $p \in f^{-1}(U)$ and $q \notin X-f^{-1}(U)$. Therefore $X$ is $\alpha$ gs- $T_{2}$.

Theorem 21. Let $f: X \rightarrow Y$ be totally $\alpha g s$-continuous injection function with $Y$ is $T_{1}$-space. If $A$ is a $\alpha g s$-connected subset of $X$, then $f(A)$ is a single point.

Proof. Let $A$ be a $\alpha$ gs-connected member of $X$ such that $f(A)$ is not a single point. Let $p$ and $q$ be distinct points of subset $A$ in $X$. As $f$ is injective $f(p)$ and $f(q)$ are distinct points of $f(A)$ in $Y$. As $Y$ is $T_{1}$-space, there exist open sets $U$ and $V$ such that $f(p) \in U, f(q) \notin U$ and $f(q) \in V, f(p) \notin V$. This indicates $p \in f^{-1}(U)$, $q \notin f^{-1}(U)$ and $q \in f^{-1}(V), p \notin f^{-1}(V)$. For the reason $f$ is totally $\alpha$ gs-continuous, implies $f^{-1}(U)$ and $f^{-1}(V)$ are proper $\alpha$ gs-clopen sets in $A$. This is contradiction to the fact that, $A$ is $\alpha$ gs-connected subset of $X$. Thus, $f(A)$ is a single point.

Definition 22. A collection $\left\{A_{i}: i \in I\right\}$ of $\alpha$ gs-clopen sets in a topological space $X$ is said to be $\alpha$ gs-clopen cover of a subset $A$ in $X$ if $A \subset \bigcup_{i \in I} A_{i}$.

Definition 23. A topological space $X$ is said to be:

1. mildly $\alpha$ gs-compact if every cover of $X$ by $\alpha$ gs-clopen sets has a finite subcover.

2. mildly countably $\alpha$ gs-compact if every countable cover of $X$ by $\alpha$ gs-clopen sets has a finite subcover.

3. mildly $\alpha$ gs-Lindelof if every cover of $X$ by $\alpha$ gs-clopen sets has a countable subcover.

4. strongly S-closed [10] if every closed cover of $X$ has a finite subcover.

Theorem 24. If $f: X \rightarrow Y$ is totally ags-continuous surjection with $X$ is mildly ags-compact, then $Y$ is strongly S-closed.

Proof. Let $\left\{V_{\alpha}: \alpha \in I\right\}$ be any closed cover of $Y$. As $f$ is totally $\alpha$ gs-continuous, $\left\{f^{-1}\left(V_{\alpha}\right): \alpha \in I\right\}$ is $\alpha$ gs-clopen cover of $X$. As $X$ is mildly $\alpha$ gs-compact, there exists a finite subset $I_{0}$ of $I$ such that $X=$ $\cup\left\{f^{-1}\left(V_{\alpha}\right): \alpha \in I_{0}\right\}$. This indicates, $Y=\cup\left\{V_{\alpha}: \alpha \in I_{0}\right\}$, which is finite subcover of $Y$. As a result $Y$ is strongly $S$-closed.

Theorem 25. Let $h: X \rightarrow Y$ be a totally $\alpha g$ s-continuous surjection. Then, the following results hold

1. If $X$ is mildly ags-compact, then $Y$ is compact.

2. If $X$ is mildly countably ags-compact, therefore $Y$ is countably compact.

3. If $X$ is mildly $\alpha g$ s-Lindelö $f$, then $Y$ is Lindelö $f$.

Proof. (1) Let $\left\{V_{\alpha}: \alpha \in I\right\}$ be any open cover of $Y$. As $h$ is totally $\alpha$ gs-continuous, $\left\{h^{-1}\left(V_{\alpha}\right): \alpha \in I\right\}$ is $\alpha$ gs-clopen cover of $X$. As $X$ is mildly $\alpha$ gs-compact, there exists a finite member $I_{0}$ of $I$ such that $X=$ $\cup\left\{f^{-1}\left(V_{\alpha}\right): \alpha \in I_{0}\right\}$. This indicates, $Y=\cup\left\{V_{\alpha}: \alpha \in I_{0}\right\}$, which is finite subcover of $Y$. Then $Y$ is compact.

(2) Let $\left\{V_{\alpha}: \alpha \in I\right\}$ be be any countable open cover of $Y$. As $h$ is totally $\alpha$ gs-continuous, $\left\{h^{-1}\left(V_{\alpha}\right): \alpha \in I\right\}$ is countable $\alpha$ gs-clopen cover of $X$. As $X$ is Mildly countably $\alpha$ gs-compact, there exists a finite subset $I_{0}$ of $I$ such that $X=\cup\left\{h^{-1}\left(V_{\alpha}\right): \alpha \in I_{0}\right\}$. This indicates, $Y=\cup\left\{V_{\alpha}: \alpha \in I_{0}\right\}$, which is finite subcover of $Y$. So $Y$ is countably compact.

(3) Lat $\left\{V_{\alpha}: \alpha \in I\right\}$ be any open cover of $Y$. As $h$ is totally $\alpha$ gs-continuous, $\left\{h^{-1}\left(V_{\alpha}\right): \alpha \in I\right\}$ is $\alpha$ gs-clopen cover of $X$. As $X$ is Mildly $\alpha$ gs-Lindelöf, there exists a finite subset $I_{0}$ of $I$ such that $X=\cup\left\{h^{-1}\left(V_{\alpha}\right): \alpha \in I_{0}\right\}$. This implies, $Y=\cup\left\{V_{\alpha}: \alpha \in I_{0}\right\}$, which is finite subcover of $Y$. Thus $Y$ is Lindelöf.

Definition 26. A topological space $X$ is said to be 
1. $\alpha$ gs-co- $T_{1}$ if for each pair of distinct points $x$ and $y$ of $X$ there exist $\alpha$ gs-clopen sets $G$ and $H$ such that $x \in G, y \notin G$ and $y \in H, x \notin H$.

2. $\alpha$ gs-co- $T_{2}$ if for each pair of distinct points $x$ and $y$ of $X$, there exist disjoint $\alpha$ gs-clopen sets $G$ and $H$ such that $x \in G$ and $y \in H$.

Theorem 27. A topological space $X$ is $\alpha$ gs-co- $T_{1}$ if and only if every singleton subset $\{x\}$ of $X$ is $\alpha g$ s-clopen set.

Proof. Let $X$ be a $\alpha$ gs-co- $T_{1}$ space and $p \in X$. Let $q \in X-\{x\}$. Then for $p \neq q$, there exists $\alpha$ gs-clopen set $U_{q}$ such that $q \in U_{q}$ and $p \notin U_{q}$. Then, $q \in U_{y} \subset X-\{x\}$. That is $X-\{x\}=\cup\left\{U_{y}: y \in X-\{x\}\right\}$, which is $\alpha$ gs-clopen set. Hence $\{x\}$ is $\alpha$ gs-clopen set.

Conversely, assume $\{x\}$ is $\alpha$ gs-clopen set for every $x \in X$. Let $x$ and $y \in X$ with $x \neq y$. Now $x \neq y$ means $y \in X-\{x\}$. Therefore $X-\{x\}$ is $\alpha$ gs-clopen set containing $y$ yet not $x$. Similarly, $X-\{y\}$ is $\alpha$ gs-clopen set containing $x$ but not $y$. Then $X$ is $\alpha$ gs-co- $T_{1}$ space.

Theorem 28. If $h: X \rightarrow Y$ is totally $\alpha g s-c o n t i n u o u s$ injection and $Y$ is $T_{1}$ then $X$ is $\alpha g s-c o-T_{1}$ space.

Proof. Let $f: X \rightarrow Y$ is totally $\alpha$ gs-continuous injection with $Y$ as $T_{1}$. For any two distinct points $x_{1}, x_{2}$ of $X$ there exists distinct points $y_{1}, y_{2}$ of $Y$ such that $y_{1}=h\left(x_{1}\right)$ with $y_{2}=h\left(x_{2}\right)$. As $Y$ is $T_{1}$-space there exist an open sets $U$ and $V$ in $Y$ such that $y_{1} \in U, y_{2} \notin U$ as well as $y_{1} \notin V, y_{2} \in V$. That is $x_{1} \in h^{-1}(U), x_{1} \notin h^{-1}(V)$ and $x_{2} \in h^{-1}(V), x_{2} \notin h^{-1}(U)$. As $h$ is totally $\alpha$ gs-continuous $h^{-1}(U), h^{-1}(V)$ are $\alpha$ gs-clopen sets in $X$. Thus, for two distinct points $x_{1}, x_{2}$ of $X$ there exist $\alpha$ gs-clopen sets $h^{-1}(U)$ and $h^{-1}(V)$ such that $x_{1} \in h^{-1}(U), x_{1} \notin$ $h^{-1}(V)$ and $x_{2} \in h^{-1}(V), x_{2} \notin h^{-1}(U)$. Therefore $X$ is $\alpha$ gs-co- $T_{1}$ space.

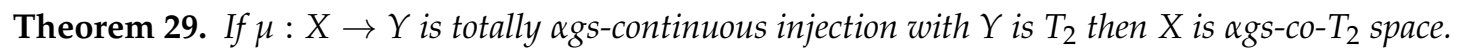

Proof. Let $\mu: X \rightarrow Y$ is totally $\alpha$ gs-continuous injection with $Y$ is $T_{2}$. For any two distinct points $x_{1}, x_{2}$ of $X$ there exist distinct points $y_{1}, y_{2}$ of $Y$ such that $y_{1}=\mu\left(x_{1}\right)$ and $y_{2}=\mu\left(x_{2}\right)$. As $Y$ is $T_{2}$ space there exist disjoint open sets $U$ and $V$ in $Y$ such that $y_{1} \in U$ and $y_{2} \in V$. That is $x_{1} \in \mu^{-1}(U)$ and $x_{2} \in \mu^{-1}(V)$. As $f$ is totally $\alpha$ gs-continuous $\mu^{-1}(U), \mu^{-1}(V)$ are $\alpha$ gs-clopen sets in $X$. Furthermore $\mu$ is injective, $\mu^{-1}(U) \cap \mu^{-1}(V)=$ $\mu^{-1}(U \cap V)=\mu^{-1}(\phi)=\phi$. Thus, for two disjoint points $x_{1}, x_{2}$ of $X$ there exist distinct $\alpha$ gs-clopen sets $\mu^{-1}(U)$ and $\mu^{-1}(V)$ such that $x_{1} \in \mu^{-1}(U)$ and $x_{2} \in \mu^{-1}(V)$. Therefore $X$ is $\alpha$ gs-co- $T_{2}$ space.

Definition 30. A function $h: X \rightarrow Y$ is said to be:

1. totally $\alpha$ gs-irresolute if preimage of a $\alpha$ gs-clopen member of $Y$ is a $\alpha$ gs-clopen subset of $X$,

2. totally pre- $\alpha$ gs-clopen if image of each $\alpha$ gs-clopen subset of $X$ is $\alpha$ gs-clopen.

Theorem 31. Let $h: X \rightarrow Y$ be surjective totally $\alpha g s$-irresolute and totally pre- $\alpha g$ s-clopen with $k: Y \rightarrow Z$ be any function. Then $k \circ h: X \rightarrow Z$ is totally $\alpha g s-c o n t i n u o u s$ if and only if $k$ is totally $\alpha g s-c o n t i n u o u s$.

Proof. The 'if' part is trivial. To claim 'only if' part, let $k \circ h: X \rightarrow Z$ be totally $\alpha$ gs-continuous furthermore, let $V$ be an open subset of $Z$. Then $(k \circ h)^{-1}(\mathrm{~V})$ is a $\alpha$ gs-clopen member of $X, h^{-1}\left(k^{-1}(V)\right)$ is $\alpha$ gs-clopen. As $h$ is totally pre- $\alpha$ gs-clopen, $h\left(h^{-1}\left(k^{-1}(V)\right)\right)$ is a $\alpha$ gs-clopen subset of $Y$. So, $k^{-1}(V)$ is $\alpha$ gs-clopen in $Y$. Thus $k$ is totally $\alpha$ gs-continuous.

Definition 32. A topological space $X$ is said to be:

1. $\alpha$ gs-co-regular if for each closed set $F$ with each point $x \notin F$, there exist disjoint $\alpha$ gs-clopen sets $U$ and $V$ such that $x \in U$ and $F \subset V$,

2. strongly $\alpha$ gs-co-regular if for every $\alpha$ gs-clopen set $F$ with a point $x \notin F$, there exist disjoint open sets $U$ and $V$ such that $x \in U$ and $F \subset V$,

3. $\alpha$ gs-co-normal if for every pair of disjoint closed sets $A$ and $B$, there exists a pair of disjoint $\alpha$ gs-clopen sets $U$ and $V$ in $X$ such that $A \subset U$ and $B \subset V$,

4. strongly $\alpha$ gs-co-normal if for each pair of disjoint $\alpha$ gs-clopen sets $A$ with $B$, there exist a pair of disjoint open sets $U$ and $V$ in $X$ such that $A \subset U$ and $B \subset V$.

Theorem 33. If $h: X \rightarrow Y$ is totally $\alpha g s-c o n t i n u o u s$, closed, injection and $Y$ is regular, then $X$ is $\alpha g s-c o-r e g u l a r$. 
Proof. Let $F$ be a closed set in $X$ and $x \notin F$. As $h$ is closed injection $h(F)$ is closed set in $Y$ such that $h(x) \notin h(F)$. Now $Y$ is regular, there exist disjoint open sets $G$ besides $H$ such that $h(x) \in G$ as well as $h(F) \subset H$. This indicates $x \in h^{-1}(G)$ with $F \subset h^{-1}(H)$. As $h$ is totally $\alpha$ gs-continuous, $h^{-1}(G)$ besides $h^{-1}(H)$ are $\alpha$ gs-clopen sets in $X$. Further $h^{-1}(G) \cap h^{-1}(H)=\phi$. Then $X$ is $\alpha$ gs -co-regular.

Theorem 34. If $\eta: X \rightarrow Y$ is totally ags-continuous, open, injection with $X$ is strongly ags-co-regular, then $Y$ is regular.

Proof. Let $P$ be a closed set in $Y$ with $y \notin P$. Take $y=\eta(x)$ being any $x \in X$, then $x \notin \eta^{-1}(P)$. As $\eta$ is totally $\theta$ gs-continuous, $\eta^{-1}(P)$ is $\alpha$ gs-clopen set in $X$, not containing $x$. As $X$ is strongly $\alpha$ gs-co-regular, there exist disjoint open sets $U$ and $V$ such that $x \in U$ and $\eta^{-1}(P) \subset V$. That is $\eta(x) \in \eta(U)$ and $P \subset \eta(V)$. As $\eta$ is open injective, $\eta(U)$ with $\eta(V)$ are disjoint open sets in $Y$. Therefore, $Y$ is regular.

Theorem 35. If $Y$ is normal and $k: X \rightarrow Y$ is totally $\alpha g s-c o n t i n u o u s$, closed, injection, then $X$ is $\alpha g s-c o-n o r m a l$.

Proof. Let $E$ and $F$ be disjoint closed sets in $Y$. As $k$ closed injection $k(E)$ as well as $k(F)$ are disjoint closed sets in $Y$. Now $Y$ is normal, there exist disjoint open sets $G$ and $H$ in order that $k(E) \subset G$ with $k(F) \subset H$. This implies $E \subset k^{-1}(G)$ with $F \subset k^{-1}(H)$. As $k$ is totally $\alpha$ gs-continuous $k^{-1}(G)$ and $k^{-1}(H)$ are $\alpha$ gs-clopen sets in $X$. Further $k^{-1}(G) \cap k^{-1}(H)=\phi$. Hence $X$ is $\alpha$ gs-co-normal.

Theorem 36. If $k: X \rightarrow Y$ is totally ags-continuous, open, injection and $X$ is strongly ags-co-normal, then $Y$ is normal.

Proof. Let us consider the disjoint closed sets $E$ and $F$ in $Y$.As $k$ is totally $\alpha$ gs-continuous, $k^{-1}(E)$ and $k^{-1}(F)$ are $\alpha$ gs-clopen sets in $X$. As $X$ is strongly $\alpha$ gs-co-normal, there exist disjoint open sets $U$ and $V$ such that $k^{-1}(E) \subset U$ and $k^{-1}(F) \subset V$. That is $E \subset k(U)$ and $F \subset f(V)$. As $k$ is open injective, $k(U)$ as well as $k(V)$ are disjoint open sets in $Y$. Therefore, $Y$ is normal.

Author Contributions: All authors contributed equally to the writing of this paper. All authors read and approved the final manuscript.

Conflicts of Interest: "The authors declare no conflict of interest."

\section{References}

[1] Jain, R. C. (1980). The role of regularly open sets in general topology (Doctoral dissertation, Ph. D. Thesis. Meerut Univ.,(Meerut, India, 1980)).

[2] Nour, T. M. (1995). Totally semi-continuous functions. Indian Journal of Pure \& Applied Mathematics, 26, 675-678.

[3] Rajamani, M., \& Viswanathan, K. (2004). On ags-closed sets in topological spaces. Acta Ciencia Indica, 30(3), 521-526.

[4] PAGE, M. H. (2015). On Almost Contra $\alpha$ GS-Continuous Functions. International Journal of Scientific and Engineering Research, 6(3), 1489-1494.

[5] Levine, N. (1963). Semi-open sets and semi-continuity in topological spaces. The American Mathematical Monthly, $70(1), 36-41$.

[6] Gene Crossley, S., \& Hildebrand, S. K. (1972). Semi-topological properties. Fundamenta Mathematicae, 74(3), 233-254.

[7] Njástad, O. (1965). On some classes of nearly open sets. Pacific journal of mathematics, 15(3), 961-970.

[8] Navavalgi, G. B., Rajamani, M., \& Viswanathan, K. (2015). On ags-Neighbourhoods. Indian Journal of Mathematics \& Mathematical Sciences, 1(2), 109-119.

[9] Navavalgi, G. B., Rajamani, M., \& Viswanathan, K. (2008). On ags-separation Axioms in topological spaces. Int J. Gen Topol, 1, 43-53.

[10] Dontchev, J. (1996). Contra-continuous functions and strongly S-closed spaces. International Journal of Mathematics and Mathematical Sciences, 19(2), 303-310. 